\section{Art Adtress}

ON THE

\section{PERIPHERAL CIRCULATION AND ITS TREATMENT.}

Delivered before the Anndal Menting of thr British Batmatogtcal and Clmmatological Socrety, 1909,

BY

SIR JAMES BARR, M.D., LL.D., F.R.C.P., F.R.S.E., SENIOR PHYSICIAN, LIVERPOOI ROYAL INFIRMARY.

THE arterioles and capillaries and venules pervade every tissue and organ of the body, and specialists in this line can never be accused of poaching on anotber man's preserves. The importance of the capillaries has been aptly described by Leonard Hill, who says:

The blood is brought into intimate relation with the tissues by diffusing through the endothelial wall of the capillaries, and this wall is of great tenuity; thereby takes place that change of material which maintains the combustion of the body and the fire of life.

It is the resistance in the arterioles and capillaries which maintains the mean or diastolic pressure in the aorta and large arteries, and any increase in this resistance has to be overcome by greater work on the part of the heart. The arterioles constitute the first line of defence, and, as I said in my Toronto address, ${ }^{1}$ it is exceedingly fortunate that there is this first line of defence created by the action of the vasomotor nerves in the small arteries and arterioles, because if this were wanting, as at present constituted we would either have to go about on all fours or constantly run the risk of fatal syncope. Moreover, the blood would gravitate into the most dependent parts, the cooling surface would be enormous, the capillary velocity would be diminished, the blood would become surcharged with $\mathrm{CO}_{2}$ and we would become cold-blooded animals. When the vasomotor nerves of a rabbit are paralysed it appears all right until you suspend it by the ears, and then it imme. diately dies. The arteriolar resistance saves us from such risks. When you get vasomotor paralysis the resistance is transferred to the capillaries, and you get a more gradual fall in the pressure gradient, the velocity of the blood in the capillaries is diminished, the pressure increased, and transudation readily takes place. In severe cases of Addison's disease in which the vasomotor nerves are deprived of their usual stimndas, the patient may not be able to assume the erect posture. The wonderful regulative mechanism of the vasomotor system enables the blood to be driven from the high-pressure main, the "aorta and its principal branches, to the organs or systems that immediately require it. Witness the great supply to the extremities during exertion, and to the abdominal viscera during digestion. Increase of pressure within an artery improves its tone and raises its co. efficient of elasticity. It is probably owing to these facts, as well as to the intervention of the vasomotor nerves, that the arteries of the lower extremities often contract when they are dependent and dilate when they are raised. This, of course, happens most readily when the arteries are healthy and the arterial blood pressure high. Under such conditions the common carotid can be felt contracted when the body is inverted. The arteries of the upper extremities obey the usual law and dilate under increased pressure. This increased tone under heightened pressure is probably a determining factor in the production of claudication of the arteries-a condition almost confined to the lower extremities, and one which is very difficult to remedy when once established. Perhaps other causal factors are excessive amount of lime salts, overaction of the suprarenal glands, and defective action of the thyroid.

The Arterioles.

It would be difficult or impossible to estimate the evervarying capacity and sectional area of the arterioles and the velocity of the blood passing through them. What we do know is that on an average a similar quantity of blood passes through a section of the arterioles to that which passes through a section of the aorta in a unit of time. The resistance in the arterioles is directly as their length, and inversely as the squaro of their cross-sections or the fourth power of the diameter, and divectly as the square of the velocity. The total sectional area of the arterioles is perhaps considerably less than that of the small arteries, and the velocity of the blood in them corre. spondingly greater. In cases of local syncope the arteri. oles shut down, and therefore the pressure and velocity in these particular vessiels fall to zero. When the arterioles are contracted we know that the energy of the blood is to a large extent converted into velocity with a corresponding diminution in the lateral pressure. If you take a cold hand, there may be a difference of $50 \mathrm{~mm}$. of mercury between the lateral pressure in the radial and that in a digital artery, but if the arterioles are dilated there is a much less abrupt fall in the pressure gradient. Moreover, in post-mortem examinations there is very seldom anything found wrong with the arterioles, except that they may be hypertrophied, but this is evidence of overaction rather than of lateral strain. The general arterial blood pressure can only be raised by the contraction of a large tract of arterioles, such as those of the splanchnic area, the skin, or muscles, and generally in health when one set is contracted another is dilated, so that a uniform mean arterial pressure is maintained. As a rule, the more contracted the arterioles the higher the arterial pressure in the arteries; and, again, this high potential in the arteries drives the blood with great velocity through the axterioles, and, of course, the greater the velocity the less the lateral pressure. A small area of arterioles, such as that of the hand, may be contracted without any effect on the general arterial pressure, and in this case the velocity of the blood in the contracted arterioles is not increased but diminished, owing to the increased friction and heightened viscosity of the blood. The blood may almost come to a standstill, and then the enexgy is largely that of pressure. When the arterioles of any local area are dilated, as at the commencement of an inflammation, or after the removal of an Esmarch's tourniquet, the whole of the capillaries are opened up and engorged with blood, and with this increased mass the pressure is raised, but the velocity is also heightened owing to the arterial potential remaining high and the resistance in the enlarged vessels being diminished. These little muscular vessels are well endowed with vasomotor nerves, and are subjected to postural variations in pressure. It is entirely due to their intervention that the capillaries are protected from the full hydrostatic effect of the blood when the body is in the erect posture. The potential increases in the arteries of the limbs when they are dependent, and if the arterioles are dilated from any cause the pressure in these little vessels and in the capillaries is much augmented, so that you may readily get petechial haemorrhages, but on the other hand, if the arterioles are much contracted, the potential in the arteries. is largely converted into kinetic energy in the arterioles and capillaries.

The capillaries through which the interchange of nutritive pabulum and gases takes place between the blood and the tissues play a most important rôle in the animal economy; they constitute a vast filter bed for conveying nutritive material and oxygen to the tissues, and for removing waste products therefrom. These little vessels are of extreme tenuity and delicacy, consisting of a single layer of endotholiam, yet they are capable of standing considerable internal pressure; they vary from about 0.5 to 1 millimetre in length, and from 7 to 13 micromillimetres in diameter. They are to a certain extent elastio, or at least they have the capacity of adapting themselves to the amount of blood which is driven through them, and they are supported by the surrounding tissues and intercelullar fluids. So numerous are these little vessels thin it would be difficult to stick the point of a needle in any rascular area without wounding one or more, bit in neurotic individuals you may wound many such vessels without drawing blood.

In cases of polycythaemia the capillaries of the body are fairly replete, but in ordinary mortals, especially in those of neurotic temperament, perhaps not a third are full at any one moment, and in a very cold extremity there may not be a twentioth of the number patent. Apply a sinapism to a very pallid skin, and you may wonder where 
all the turgid capillaries have sprung from. By warm baths, friction, and general massage the arterioles are relexed, the capillaries opened up, and hence there is a general fall in the arterial blojd pressure. The energy in the capillariss is derived from that in the arteries and arterioles, and is a compound of lateral pressure and velocity. You will find all these problems discussed in my Toronto address, and I am here only concerned with the practical application of our science. The more dilated the arterioles and the more vascular the area, caeteris paribus, the greater the pressure and the less the velocity, and vice versa. The lateral pressure depends on the statical condition of the blood, and just in proportion as you introduce movement you convert the force of pressure into that of velocity. If you wish to drive a certain quantity of fluid through a set of tabes, the velocity will depend on the force of propulsion minus the obstruction to the outflow, with the inertia or viscosity of the blood (there is no fluid perfectly mobile) and the friction of the tubes; and the lateral pressure will increase as the outflow. is obstructed-in short, as the statical condition is maintained.

\section{The Caprlatary Pregsure.}

For estimating the pressure in the capillaries, perhaps the most convenient method is that of von Kries. I use three sizes of glass plates, measuring 20,100 , and $400 \mathrm{sq} . \mathrm{mm}$., so a gram pressure on each of these plates represents respectively 50,10 , and $2.5 \mathrm{~mm}$. of $\mathrm{H}_{2} \mathrm{O}$. The pressure stands in direct relation to the freedom of the inflow and the obstruction to the outflow. When the body is erect the depeudent capillaries are largely protected from the brunt of the increased hydrostatic pressure by the intervention of the arterioles, but, notwithstanding this intervention, the pressure in the capillaries of an immobilized foot when the subject is standing, becomes very great, but, if you move the foot, the capillaries are compressed, the blood is driven onwards and the statical pressure is converted into velocity. The increased poten tial in the dependent arteries is thus partly converted into pressure and partly into velocity in the capillaries, and the velocity is further heightened by movement. The inver-sion of the body for a comparatively short time each day - over a lengthened period should be sufficient to give rise to marked arterio-sclerosis of the cerebral vessels owing to increased statical pressure, because in the brain you could - not introduce movement to hurry on the circulation. Moral, "Keep your head above water." All occupations which involve much standing or sitting must be injurious to the vessels of the lower extremities, hence such indi. viduals should be encouraged to keep their lower limbs as restless as possible. Kentish fire relieves the monotony when you are listening to a wearisome speaker. Probably if an audience in church could indulge in this form of recreation their brains would be better supplied with blood, there would not be so many asleep, the hypnotic influence of the sermon would not be so effective, and possibly the Nonconformist conscience would not be so paralysed as it usually is to all extraneous influences for good.

All agents, such as dry heat, warm baths, and to a less extent massage and passive exercises which relax the arterioles; increase the freedom of flow into the capillaries and raise the lateral pressure in them and lower the arterial pressure. In proportion as the general arteriolar resistance is relaxed the resistance to the output from the heart is transferred from the arterioles to the second line of defence. This increases the bed of the arterial river and so lessens the resistance to the systolic output, but it -does not necessarily lessen the total work of the heart, because the venous pressure is also raised and with it the diastolic tension in the ventricles, and consequently the systolic output. If the arteries be at all rigid, and not capable of adapting themselves to the altered conditions there may be a great waste in the cardiac energy. Hence the injudicious use of baths and vasomotor relaxants in the aged very readily gives rise to weariness, languor, faintness, and sickness. Those who think that warm baths are a panacea for all ills to which flesh is heir should be careful when administering them to those with sclerotic arteries to cloak their ignorance by keeping the patients afterwards for a long time in the recumbent posture until the arteries and arterioles have had time to regain their tone. After a warm bath, dry or moist, a cold or cool douche is usually a valuable adjuvant.

The Velocity of the Blood in the Capillarirs.

In textbooks of physiology the capillary velocity is usually put down as 0.2 to $0.75 \mathrm{~mm}$. per second, but in my opinion this is much too low an estimate of the average velocity, and I have said that anyone with a capillary velocity at the level of the heart which physiologists set down as normal might appropriately take up the refrain. "The hour of - my departure's come." My method of calculating the capillary velocity is to take a glass rod $10 \mathrm{~mm}$. in diameter and with the flat end compress the blood out of the capillaries, then . with a stop-watch recording fifths of a second I time the period of the return of the blood. If you divide the radius of the rod $(5 \mathrm{~mm}$.) by the time, you get the apparent velocity per second. When fluid is circulating in a capillary tube the axial velocity is double the mean velocity. Now, the erythrocytes travel in the axis, but as they occupy at least four. fifths of the lumen of the vessel the mean must be about 0.8 of the apparent velocity. Under this method of calculation I have observed the capillary velccity to vary from about $0.4 \mathrm{~mm}$. per second when the patient is dying to about $20 \mathrm{~mm}$. per second in the great toe when the patient is standing and there is very high arterial potential. We know that in the large capillary bed the current is chiefly onwards to the veins, but in many of the capillaries the current is often reversed, and this lowers the mean onward velocity, but even if you half my estimates you are still a long way above the estimates of physiologists. In making your observations you must select some spot where there is a network of capillaries which you can c smpletely empty, such as those in the back of the hand or finger, and you must also choose a spot where the return current flows from all parts of the circumference to the centre of your compressed area. You cannot estimate the velocity in the palmar surface of the finger end, because the small arterioles run up to the papillae at right angles to the surface, and when the blood is driven out it returns to all parts of the compressed area at the same time. For the sake of uniformity and comparison it is well to estimate the velocity in the hand, or other part, at the level of the heart, because the velocity is much increased by raising the potential in the arteries; you can often double the capillary velocity in the finger by lowering it 300 or $400 \mathrm{~mm}$. below the heart level. The velocity of the blood in the capillaries stands to that in the arterioles inversely as the square of their cross sections, but as we do not know the sectional area of the arterioles there will be some difficulty in arriving at that of the capillaries. The velocity in these vessels depends on the arterial potential, minus the obstruction to the outflow with the friction against the tubes and the viscosity of the blood. I find the capillary velocity a valuable aid in forming an opinion as to the viscosity of the blood; if at the level of the heart-except, perhaps, in cases of polycythaemia - the velocity be high, you may conclude, without drawing a drop of blood, that the viscosity is not abnormal.

If there be a great difference between the systolic pressure in the aorta and the resistance, such as occurs in free aortic regurgitation or very advanced arteriosclerotic changes, the arterial wave may be carried to the capillaries and give rise to pulsation in these vessels. This implies great waste of cardiac energy, and under such circumstances vasomotor relaxants must work mischief; yet many of your fraternity ignorantly prescribe their favourite baths without any knowledge of or regard for physical consequences. Vasomotor tonics and such agents as diminish the lime salts and improve the muscular tone constitute the best treatment.

Any obstruction to the outflow from the capillaries diminishes the velocity in them. Hence the congestive treatment of joints and other local lesions which is now known as the Bier treatment, but which was long ago carried out by the late Hugh Owen Thomas of Liverpool, while it lessens the velocity, it increases the statical pressure and transudation from the capillaries to the tissues. The extra-capillary pressure is about one-fourth of that within the vessels, and largely contributes to the onward movement of the lymph; this is also aided by muscular contractions, in the same way as assistance is thus given 
to the capillary circulation. The Bier treatment retards the circulation of the lymph and blood and increases transudation, while massaye has the opposite effects. Dr. Barry at the beninning of last century advocated the use of the suction pump to prevent the introduction to the system of the venom of snake-bite.

\section{The Viscosity of thr Blood.}

The viscosity varies greatly, and is no doubt the great cause of the resistance in the capillaries. Normally it is about five times that of distilled water. This subjecr has been very ably investigated by Dr. John $H$. Watson and Dr. A. du Pre Denning, who have shown how an increase in the number of corpuscles great]y increases the viscosity. It is diminished by fever and increased by carbonic acid and I have shown that when the capillary velocity falls to $1 \mathrm{~mm}$. to the second or less the blood becomes surcharged with $\mathrm{CO}_{2}$

The viscosity is much increased by the salts of calcium, magnesium, manganese, and strontium, and hence these salts lessen transudation, and are thus useful in cases of orthostatic albuminuria, eczema, and urticaria. It is diminished by citric and phosphoric acids, the salts of potassium, ammonium, and sodium, thyroid and iodine. An increase in viscosity produces a most definite rise in the internal resistance, but we must remember that the living capillaries are more or less elastic, and offer much less resistance to the flow of blood than would be caused by rigid tubes of the same diameter. Moreover, it has been shown by Watson and Denning that " a given increase of pressure exerts a much greater accelerating effect on the rate of flow through tubes of fine calibre than through tubes of wide bore." This is corroborated by my observations on the great increase in the velocily in the capillaries produced by an increase in the arterial potential. By lessening the viscosity of the blood you lower the arterial resistance and diminish the work the heart. In many cases the viscosity is too low, and this is, as a rule, easily remedied by the salts of calcium and magnesium.

\section{The Interchange of Materials throdgh the} Capillary WaLis.

There are some physiologists who would raise the endothelial cells of the capillary walls to the high level of secreting structures; not that they have any evidence, either from analogy or otherwise, in support of such a contention, but simply because they think that the physical properties of diffusion, osmosis, and filtration cannot account for the phenomena. They hold that the capillary pressure is low, and is more than counterbalanced by the endosmotic equivalent of the albumen and salts in the blood. When they recognize the enormous variations which take place in the pressure and velocity of the blood in the capillaries, they will have less difficulty in admitting the problem of filtration as applicable to the capillary wall. Moreover, the capillary walls do not form a semipermeable membrane, but are just as easily permeated, caeteris paribus, in one direction as in the other In a network of capillaries the pressure must necessarily be higher in the efferent or distributing vessels connected with the arterioles than it is in the afferent or collecting tubules which unite to form the venules. You can thus have filtration and absorption going on side by side, just as in a hole in the wall divided by a midriff you can have strong currents of air flowing side by side in opposite directions. The fall in the pressure gradient in the capillaries must be fairly uniform from arteriole to venule; but, as there is usually a considerable fall from the capillaries to the venules, there must be a corresponding difference between the efferent and the afferent capillaries, and thus transudation and absorption in dif ferent parts of the same network can be readily explained. In the intestinal capillaries the larger and deeper vessels supply the secretory structures and the smaller and more superticial vessels are the absorbants.

Physiologists usually infer that some healthy capillaries are more permeable than others, and here, again, they are content with inferences rather than proofs, because they find a greater transudation, say, from the capillaries of the liver than from those of the foot; but they forget that in the foot, compared with the liver, the capillary bed to each arteriole is much less, the velocity is enormously greater, and when the foot is moving the pressure may be less, and the exuded lymph is moved on as quickly as it is formed. Surgeons often find the permeability of the capillaries of an immobilized limb too great to their liking. In filtration the concentration of the filtrate is greater at lower than at higher pressures, bence the lymph from the liver capillaries often contains 6 to 8 per cent. of albumen. In alcoholics the arterial blood pressure falls, the diastolic pressure in the beart rises, the right ventricle gets dilated and tricuspid valve incompetent, and the blood is thus dammed back in the lirer. From the fall in the arterial pressure, the pressure in the portal vein also falls, and consequently there is a fall both in the velocity and filtra. tion pressure in the portal capillaries; this gives rise to a concentrated transudate both in albumen and leucocytes which soon leads to a multilobular cirrhosis. This obstructs the portal circulation, which raises the pressure in the intestinal capillaries and gives rise to increased filtra. tion from them. The liver becomes of a deep purple colour when the circulation in it is sluggish from any cause, owing to the blood being surcharged with $\mathrm{CO}_{2}$. The indications for treatment in these cases are: Stop the alcohol, lessen the input of fluid, and raise the arterial pressure. An intestinal antiseptic and a colon bacillus vaccine are often useful. There is no use in washing them out with any mineral spring, unless it be some concentrated saline which runs through the intestinal tract without being absorbed. In my opinion a dose of Epsom or Glauber salts in a smal quantity of water is better than any imported saline.

In 1904 Dr. George Oliver published his admirable work on the tissue lymph circulation. He showed that during the first hour of digestion there was a rise in the blood pressure-arterial, capillary, and venous-with a flow of lymph into the tissues. His conclusions were:

1. That the food constituents themselves (proteids, fats, and carbohydrates) do not possess the power of starting the mechanism by which lymph is dispensed to the tissues through the body.

2. That Nature, however, associates with our foodstuffs smal quantities of very active substances which bring into play that mechanism, though these substances themselves are practically devoid of food value, and that man frequently increases this natural $\mathrm{rmph}$ by the use of salt, and beverages containing bodies which also incite the flow of lymph. Such bodies a uric acid, creatin, creatinin, xanthin, glycogen, and sodinm chloride perform an important function in nutrition for durin digestion the act a distributors of lymph to all the tiogued digestion they act as distributors of lymph to all the tissues-an office which the nutrient constituents themselves
and carbohydrates) are incapable of discharging.

He associated the action of these lymphagogues with a rise in the capillary pressure, and not, like Heidenhain, to any specific excitation of the secretory activities of the endotbelial cells. He also accepted the dictum of Starling and other physiologiste, that an increased lymph concen tration was due to increased permeability of the capillaries, and here I differ from them. No doubt capillaries damaged by inflammation or by any solution of continuity will readily permit of the exudation of albumen, but the variations in the quantity and concentration of lymph from healthy capillaries is readily accounted for by varia. tions in the capillary pressure and velocity. When there is great pressure and high velocity, there may be great transudation of thin lymph from the efferent or distributing capillaries, and an equally rapid absorption by the collecting or afferent capillaries. When the pressure and velocity are low, as in the liver, you get a concentrated lymph and practically no absorption. The interchange of gases takes place by diffusion.

\section{The Vendles.}

These little veins are smooth, capacious vessels which offer little or no resistance to the circulating blood. They are slightly elastic and attain their maximum distension at a low internal pressure. They are contracted by cold and mechanical stimulation and dilated by heat. The energy of the blood in them is a compound of pressure and velocity, and depends on (1) the energy transmitted through the capillaries, (2) the hydrostatic pressure, and (3) the aspiration of the thorax. There is a gradual fall of pressure from the venule to the right auricle. Although the circulation of the blood has been known for over 300 years, I believe it was left to me to discover that the venous blood runs more quickly up the hill than it does down it, and this is due to the increased energy in the 
arteries and capillaries in a dependent limb. We will now consider the principal local circulations.

The Splanchic Arra.

We have already referred to the circulation in the liver. The arterioles of the digestive tract are very muscular and well supplied with vasomotor nerves of the constrictor type. These nerves and vessels are the chief regulators of the arterial blood pressure. The capillaries are very fine, and form an exceedingly close network. The resistance to the outflow is very slight, except in cases of portal obstruction; hence the velocity is usually great and the lateral pressure slight. This is just what would be expected in vessels where absorption is an even more important function than secretion. The bright pinkish hue affords a marked contrast to the dark purple liver where the circulation is sluggish.

When you get complete vascmotor paralysis, as in severe shock, the greater part of the blood of the body may drain into the splanchnio area. From this there may be all degrees, down to a slight paresis, which has been described by Albert Abrams under the title "cardio-splanchnic paresis." Witness the pale, pasty f flabby youths who are often treated for anaemia, but whose haemoglobin is not deficient, and when they are laid flat the colour of the face at once improves. These cases are more frequently young males than females, and I believe in the majority of cases the paralysant is nicotine. There is occasionally a deficiency of lime salts and slight albuminuria. These cases are closely allied to orthostatic albuminuria, or the so-called albuminuria of adolescence, where, in addition to cardio-splanchnic paresis, there is always a deficiency of lime salts. From the very time that this condition began to attract attention I taught that it was entirely circulatory in origin, and was quite independent of any disease of the kidneys. When resting the patient should be quite horizontal, with a low head. When erect he should not stand or loll about, but take plenty of exercise and wear a tight abdominal belt. He should get the glycero-phosphate or lactate of lime, and, when a purgative is required, sulphate of magnesium. He should be on a liberal dry diet, consisting of beef, mutton, chicken or fish, vegetables, milk puddings, a little tea, coffee or buttermilk, little or no salt, and no acids. In all these cases tobacco and alcohol should be forbidden.

In these cases there is often an attempt on the part of Nature to compensate for the cardio-splanchnic paresis by contraction of the arterioles of the skin and muscles, and you can roll the arteries of the extremities, like thick elastic tubing, under your fingers. This raises the general arterial. pressure, but it works mischief by further congesting the splanchnic area. There is often epistaxis, and there may be slight haemoptysis without any disease of the lungs. General massage, the Aix douche, or Nauheim baths are beneficial ; but whatever course of treatment be adopted, physical exercises must not be neglected, and certainly the patient must not be drenched with mineral waters; all such are abominable in these cases.

Each infective process requires its own specific treatment, but in all such cases it is well to keep the bowels open and as aseptic as possible. Sour buttermilk is a good antiseptic for the intestinal tract.

The Arterioles and Capillarizs of the Kidinys.

We have just seen how the deficiency of lime salts in the blood, and the increased pressure in the abdominal aorta and inferior vena cava gives rise to orthostatic albuminuria when there is paresis of the splanchnic vessels, but these conditions are quite different in granular kidneys. Here the muscular arterioles, which are well supplied with vaso-constrictor nerves, are contracted and protect the capillaries of the glomeruli from any excessive pressure, but the high potential in the aorta drives the blood through the arterioles with great velocity, and this energy is expended in the Malpighian corpuscles, partly as pressure and largely as velocity; hence we get a large dilute filtrate. The glomeruli are further protected by the endothelial lining of Bowman's capsule, which in these cases becomes much thickened, and is not easily permeable to the large molecules of albumen, but readily allows the transudation of water and salts. There is nocturnal Jiuresis, because in the horizontal posture, although there is a slight fall in the general arterial pressure, the arteries of the kidneys are dilated and the total amount of blood circulating through them increased. In these cases the lime salts are usually excessive, and hence milk is not a suitable diet, though many medical men think it is the only proper diet in all diseases of the kidneys. The nitrogenous elements must be cut down and salt almost eliminated from the diet. The patient can have plenty of hydrocarbons, carbohydrates, fruit and vegetables, except such as contain oxalic acid. Medicinally, he should have the benzoates, the citrates; and any decalcifying agents.

In acute inflammatory disturbance of the kidneys the velocity in the capillaries is diminished and the lateral pressure relatively high; hence we get a concentrated filtrate. In healthy capillaries about a fourth, or less, of their pressure is transmitted directly to the surrounding tissues in which they are imbedded, but in the large congested, chocolate-coloured kidneys, when the capsule is atretched to its utmost capacity and the kidneys are nearly twice their normal weight, nearly the whole of the arterial and capillary pressure is transmitted and stops all secre. tion. The only salvation for such kidneys and their possessor is to freely incise the capsules and kidneys, as was ably and persistently advocated by the late Reginald Harrison. When the tension is relieved the secretion at once starts. A similar condition is often seen: in cases of adenitis.

In acute tubular nephritis the lime salts are not, as a rule, excessive; hence, from this point of view, milk is admissible, and it has also the advantage of being poor in chlorides, but it is far too nitrogenous to be commended. I prefer fruit and carbohydrates, on which the patient can subsist for a long time. Medicinally, the following drugs are useful: Antimony, the benzoates, the citrates, the acid tartrate of potassium, saline purgatives, hot-air baths.

The Arterioles and Capillaries of the Skin.

The arterioles are very muscular and well supplied with vasomotor nerves, chiefly of the constrictor type; frequently they are so contracted that the capillaries are almost empty, and in cases of local syncope quite empty, and the pressure and velocity fall to zero. These vessels are reciprocal to those of the splanchnic area. Usually when one set contracts the other dilates, and thus the mean arterial pressure is regulated; and they are also largely concerned in regulating the temperature of the body and the watery exhalations from the skin. When there is general vasomotor paralysis the blood travels in the direction of least resistance and largely accumulates in the splanchnic area; but if in addition you have general plethora it also stagnates in the extremities and other dependent parts and gives rise to general oedema. When there is merely local vasomotor paresis, such as that produced by a sinapism, the capillaries are engorged and the pressure and velocity increased. Witness also the effects following the application and removal of an Esmarch's tourniquet. Of this method of treatment Dr. William Ewart has made good use in rheumatoid arthritis.

The capillaries form an exceedingly close network in the corium and send loops up into the papillae, and the pressure and velocity vary enormously. After a liberal meal-especially one containing ingredients which dilate the arterioles, raise the diastolic arterial pressure, and thus provide an abundant supply of blood to, with increased pressure in, the capillaries-there is a free outpouring of lymph. In my opinion, a good deal of the work which has been done in the so-called digestion leucocytosis has been rendered worthless by the work of George Oliver on the tissue-lymph circulation. Those observations were made on the blood obtained from a prick of the finger, and this consists of a mixture of blood and lymph. The white cells are increased-the increase being in the lymphocytes-in proportion to the dilution with lymph, and the red cells are proportionately diminished. If the lymph be compressed out of the tissues and then a drop of pure capillary blood be obtained, there will be found in it an increase of red cells, and the white cells will be much less than in the mixed blood, and scarcely any more than would be obtained by an ordinary puncture three or four hours later, when the lymph had been reabsorbed. This socalled digestion leucocytosis is therefore no evidence of any increase of white corpuscles in the circulating blood, 
but merely that lymph has transuded from the vessels, and in this lymph there. is a considerable number of Aymphoagtes, probably obtained from the tissues as well as from the capillaries. There is no donbt a digestion leucocytosis, but it is not what has been usually described.

\section{Baths.}

These axterioles and capillaries offer an extensive area for the practice of the bath physician; if he cannot here cultivate fresh fields and pastures new, he is not worth his adt. A little washing does every one good, especially those who only take an annual tub. The nature of the bath depends on the objects which you want to aocom. plish. Heat relaxes and cold contracts. the arterioles, effleurage causes dilatation, bat pétrissage and tapotement rather contract the skin arterioles and dilate those of the muscles. The light or hot-air bath-if not overdonefollowed by gentle massage and a cool douche, has often a very stimulating effect. The warm alkaline bath has a sedative, and the carbonic acid bath a stimulating, effect on the nerve endings. In the Nauheim bath the increased specific gravity has a primary effect of raising the peripheral resistance, which is afterwards followed by reaction, and the effervescence has a stimulating effect, but it is a piece of absurd twaddle and unreasoning credulity to attribute any specific effects to the chloride of calcium; fortunately this salt is not absorbed, otherwise it might work irreparable mischief. Baths of all ikinds are, think, powerful agents for good or evil, and should be used with care and discrimination. In advanced arteriosclerotic changes they do good in great moderation, but it is extremely easy to work mischief. There is no use in telling your patients, as is often done, that they will feel the benefit of the baths when they return home; this, in my opinion, is equivalent to saying that the patient would have been better without the baths as administered at your particular spa. Any beneficial effect should be observed under administration. I do not like this hope deferred, and I think under such circumstances the patient on his return home should thank God for his escape.

The Arteriolrs and Capiutaries of THe Mosches.

The arterioles are supplied with vaso.dilator nerves and are thus reciprocal to those of the splanchnic area. The capillaries are arranged in a fine longitudinal network, from which lymph readily transudes. Dr. George Oliver has shown that during the height of the digestive flow of lymph tension exercises of the muscles do not further xaise the arterial pressure. The lymph in the limbs is not concentrated and is readily absorbed. Sir Lauder Brunton and Dr. F. W. Tunnicliffe showed that after the cessation of muscular contraction the intermuscular arterioles dilate and thus lessen the resistance. Healthy muscular exercise is necessary for the maintenance of a vigorous circulation, and when such exercise cannot be taken, we should use such substitutes as massage, passive exercises, and douches.

The Cerebral Vessels.

As the circulation in the brain is carried on in a closed box, and owing to the atmospheric pressure, the total amount of blood varies very little. The arteries and arterioles are thin-walled, the inner coat is well formed, there is a moderate amount of muscle fibre in the middle coat, the external coat is rather defective, but it is strengthened by a lymphatic sheath. The presence of vasomotor nerves is not generally acknowledged, but Dr. Alexander Morison says that he discovered them. Owing to stress and strain these vessels are liable to atheromatous and calcareous degeneration, and in the smaller vessels there are frequently miliary aneurysms. The capillaries are small, short, well-supported vessels, which seem to be able to bear a considerable amount of strain. When the body is inverted-not a desirable method of treatment for any affection, except that of syncope-the carotid arteries contract down to about half their usual calibre, and thus the quantity of arterial blood is lessened, but its kinetic energy increased. The arteries are subjected to great strain from higher potential, but in the capillaries this is largely converted into velocity. The cerebro-spinal fluid, which represents the lymph, is very deficient in proteid, from which we may infer that under ordinary circumstances the capillary velocity is relatively great and pressure slight. In cases of meningitis the proteid in the fluid is increased. In alcoholic plethoric inctividuals. there is frequently a deficiency in lime salts, transudation takes place into the ventricles, and so serous apoplexy is not such an uncommon erent as textbooks make out. In cases of high arterial pressure the arteries are very liable to atheromatous and calcareous. degeneration; such cases are often terminated by the rupture of a degenerate vessel or miliary aneurysm.

The treatment is that of the cause; frequently a timels free blood-letting saves the patient. Turkish baths are especially to be condemned in such cases, and other forms should only be used with great circumspection.

\section{The Coronary Vessers.}

The arteries and arterioles which supply the heart closely resemble the cerebral vessels in being thin-walled, rather deficient in muscular fibre, and in having very few, if any, vasomotor nerves. They are also exceedingly prone to atheromatous and calcareous degeneration of the intima. Newell Martin, Roy and Adami, and Alexander Morison found some evidence of vasomotor nerves, but, on the other hand, Schäfer, Dixon, Brodie, and Elliott have failed to get any response to adrenalin, which acts on all muscular fibre innervated by the sympathetic. Recently Dr. W. H. Harvey has found that the pituitary gland contracts these vessels and causes atheroma in them. These arteries are terminal, and this would seem to be one of the very few mistakes which Nature has made, as we might be inclined to think that here, if anywhere, a free anastomosis is desirable. We must, however, remember that the two sides of the heart are two distinct organs only linked together for convenience and mutual support. Perhaps, also, Nature is more concerned with the species than with the individual, and to her the loss of a person from a blocked coronary artery is a matter of no great moment. The terminal portions of the arteries which are imbedded in the muscle remain fairly healthy as a rule, so when a coronary is blocked the heart may be supplied with blood from the venous side. In cases of tricuspid stenosis the coronary veins are much dilated, and form regular sinuses in the cardiac muscles.

I have dealt with the treatment of chronic degenerative lesions of the heart and with the pulmonic circulation in previous addresses.

I have not spoken about the climate because in this country we have got to take it as we find it, but, such as it is, if it were not for the greed of the hotel proprietors, it could be had at a very reasonable price. Moreover, there are the advantages that the atmosphere is frequently washed, the sewers flushed, and the sanitation, as a rule, perfect.

1 British Medical JournaL, 1906, vol. ii, p. 401.

\section{THE CRESCENDO M.URMUR OF MITRAL STENOSIS.}

By E. M. BROCKBANK, M.D.(VICT.), F.R.C.P., HONORARY ASSISTANT PHYSICIAN, ROYAL INFIRMARY, MANCHESTER.

IN the extensive investigation of the physical signs and symptoms of cardio-vascular disease which is going on at the present day the rhythm of the crescendo murmur of $a \cdot v$ stenosis is apparently playing a very important part. Theories to explain perverted action of the heart are being promulgated; interpretations of jugular pulse tracings, normal or abnormal, deductions for prognosis, and indications for treatment are being made, books are written, and papers published, all being based in part or wholly on the supposed fact that the crescendo murmur of $a \cdot v$ stenosis is presystolic or auricular systolic in rhythm. As I have been of the opinion from 1897, when I first wrote on the subject, and am more strongly of the same mind now, that this theory has not only never been proved, but that it is fallacious, I think it worth while to remind observers of the alternative view, and to draw attention to some recent work, because it provides most valuable evidence as to the actual time of onset of this characteristic murmur.

In the first place, however, I should like to review the main theories concerning the rhythm of the crescendo murmur of mitral stenosis (for convenionce I will speak of 